\title{
Completely laparoscopic extraperigastric lymph node dissection for gastric malignancies located in the middle or lower third of the stomach
}

\author{
Ichiro Uyama, Atsushi Sugioka, Junko Fujita, Yoshiyuki Komori, Hideo Matsui, Ryohei Soga, \\ Atsushi Wakayama, Kitchiro Oкamoto, Akihiro Ohyama, and Akitake Hasumi \\ Department of Surgery, Fujita Health University, School of Medicine, 1-98 Dengakugakubo, Kutsukake-cho, Toyoake, Aichi 470-1192, Japan
}

\begin{abstract}
:
Dissection of the extraperigastric lymph nodes is necessary in most submucosal gastric cancers. Laparoscopy-assisted gastrectomy with extraperigastric lymph node dissection via minilaparotomy has been performed, but, to our knowledge, completely laparoscopic extraperigastric lymph node dissection has never been reported. We successfully performed completely laparoscopic distal gastrectomy with extraperigastric lymph node dissection in 12 patients, of whom 11 had early gastric cancer and 1 had malignant lymphoma. This surgery is technically feasible, has an acceptable complication rate, and a curability similar to that with open surgery.
\end{abstract}

Key words: completely laparoscopic surgery, lymph node dissection, gastric cancer

\section{Introduction}

Since laparoscopy-assisted distal gastrectomy for early gastric cancer was first performed in March 1992 by Kitano et al. [1], it has been used in the treatment of gastric cancer at multiple centers [2-6]. However, unlike its use in colon carcinoma, completely laparoscopic radical lymph node dissection has not been reported with gastric cancer. This distinction can be attributed to the anatomy of the stomach region, which is complex compared with the colon and which poses considerable technical challenges for surgeons.

Herein, we describe the technique and initial results for completely laparoscopic extraperigastiric lymph node dissection in distal gastrectomy.

Offprint requests to: I. Uyama

Received for publication on June 15, 1999; accepted on Aug. 18, 1999

\section{Definition of regional lymph nodes}

The numbers and groups of the regional lymph nodes were defined according to the general rules of the Japanese Research Society for Gastric Cancer [7]. We routinely remove both group 1 and group 2 lymph nodes in patients with early gastric cancer located in the middle or lower third of the stomach. The group 1 lymph nodes consist of the right cardial lymph nodes (no. 1), the lymph nodes along the lesser curvature (no. 3 ), the lymph nodes along the left gastroepiploic vessels (no. $4 \mathrm{sb}$ ), the lymph nodes along the right gastroepiploic vessels (no. 4d), the suprapyloric lymph nodes (no. 5), and the infrapyloric lymph nodes (no. 6). The group 2 lymph nodes consist of the lymph nodes along the left gastric artery (no. 7), the lymph nodes along the common hepatic artery (no. 8a), the lymph nodes around the celiac artery (no. 9), and the lymph nodes along the proximal splenic artery (no. 11p). Although the lymph nodes in the hepatoduodenal ligament (along the hepatic artery) (no. 12a) and along the superior mesenteric vein $(14 \mathrm{v})$ are classified as group 2 in the new Japanese Classification of Gastric Cancinoma [8], these lymph nodes were not removed in this series.

\section{Selection of patients and preoperative preparation}

The indications for laparoscopic distal gastrectomy were: (a) tumor located in the middle or lower third of the stomach, (b) depth of tumor invasion confined to the submucosal layer (T1), (c) no evidence of lymph node metastasis (N0) or metastasis confined to group 1 nodes (N1), and (d) tumor unsuitable for either endoscopic mucosal resection or laparoscopic local resection. Neither tumor size nor histologic type were among the selection criteria. Patients with medical conditions which precluded general anesthesia were not enrolled in this study. A history of upper abdominal surgery was 
not an absolute contraindication. Twelve patients were enrolled in this study, 11 with early gastric cancer and 1 with malignant lymphoma.

Preoperatively, autologous blood $(400 \mathrm{ml})$ was banked. Each lesion was marked endoscopically with four clips (MD-59; Olympus Optical, Tokyo, Japan), using a clip applier (HX-4U; Olympus).

\section{Operating room setup}

The patient, under general anesthesia, was placed in the supine position with the legs apart. A 20-degree headup tilt was employed in order to prevent the transverse colon or small intestine from visually compromising the operative field. The surgeon stood on the patient's right, with the first assistant on the patient's left and the camera operator between the legs. Two video monitors were placed over the patient's shoulders.

\section{Surgical technique}

After pneumoperitoneum was established using the open technique, five to six ports $(12 \mathrm{~mm}$ in diameter) were placed. A flexible electrolaparoscope (Fujinon, Tokyo, Japan) was introduced through the infraumbilical port.

The procedure began with the dissection of no. $4 d$, and the no. $4 \mathrm{sb}$ lymph nodes. The gastrocolic ligament was divided proximally about $4 \mathrm{~cm}$ from the epiploic arcade toward the lower pole of the spleen, using ultrasonic shears (Laparosonic Coagulating Shears: LCS; Ethicon, Cincinnati, OH, USA). The roots of the left gastroepiploic vessels were exposed using an ultrasonic aspirator (Ultrasonic Surgical Unit: USU; Olympus Optical, Tokyo, Japan), and were divided with double clips (Fig. 1). The no. 4sb lymph nodes were divided from the greater curvature using the LCS. The division of the gastrocolic ligament was continued distally toward the pylorus using the LCS. The roots of the right gastroepiploic vessels were exposed, using the USU, and were divided with double clips. The no. 6 lymph nodes were dissected from the duodenum using the LCS (Fig. 2). The right gastric artery was exposed and divided at its origin with double clips, allowing the dissection of the no. 5 lymph nodes (Fig. 3). The duodenum was transected $1 \mathrm{~cm}$ distal to the pylorus, using an endoscopic stapling device.

Caudal retraction of the pancreas with the Endoretractor II (United States Surgical, Norwalk, CT, USA.) facilitated the exposure of the common hepatic artery, splenic artery, and celiac artery. The no. 8a, 9, and $11 \mathrm{p}$ lymph nodes were dissected along each artery, using the USU and LCS (Fig. 4). The left gastric vein

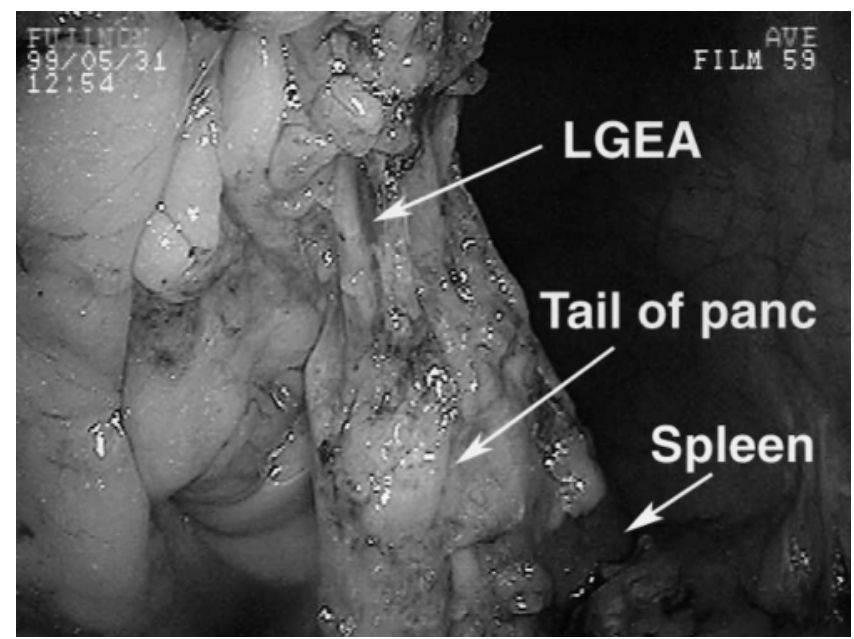

Fig. 1. Exposure and division of the left gastroepiploic artery. $L G E A$, Left gastroepiploic artery; tail of panc, tail of the pancreas

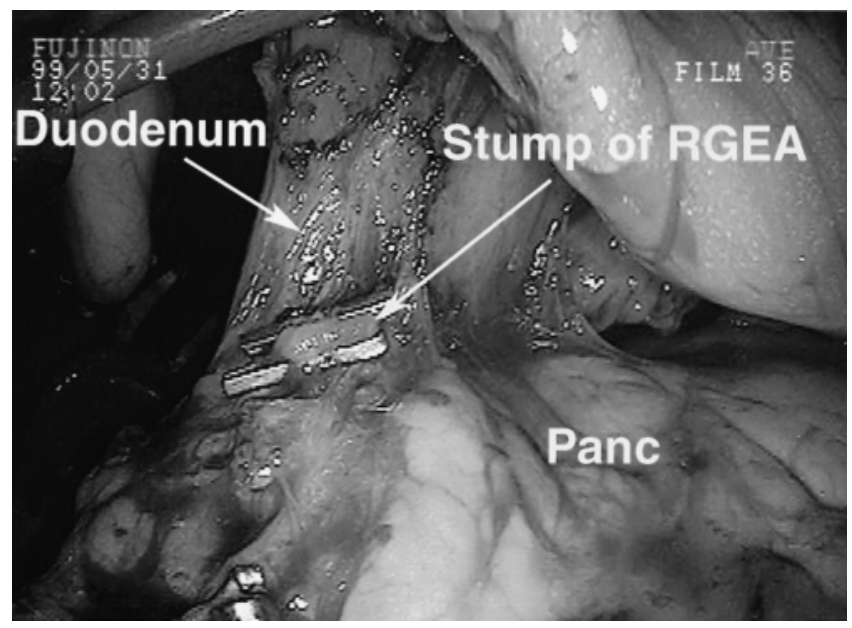

Fig. 2. Dissection of the infrapyloric lymph node. $R G E A$, Right gastroepiploic artery; panc, pancreas

was divided, and the root of the left gastric artery was exposed and divided with double clips, allowing the dissection of the no. 7 lymph nodes (Fig. 5). Further dissection of the no. 9 lymph nodes was added proximally (Fig. 6).

The no. 1 lymph nodes were dissected along with skeletonization of the upper third of the lesser curvature (Fig. 7). The resection line was determined using intraoperative gastroendoscopy, and the preoperatively applied intragastric clips confirmed the location of the tumor. The stomach was transected using the endoscopic stapling devices, and an en-bloc resection of the stomach and extraperigastric lymph nodes was completed. The resected stomach and lymph nodes were 


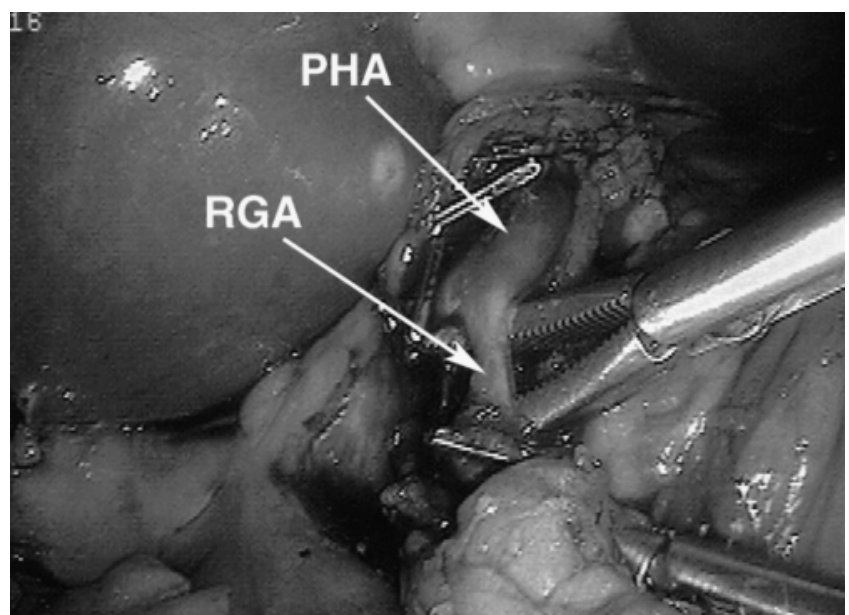

Fig. 3. Exposure and division of the right gastric artery. (RGA) PHA, Proper hepatic artery

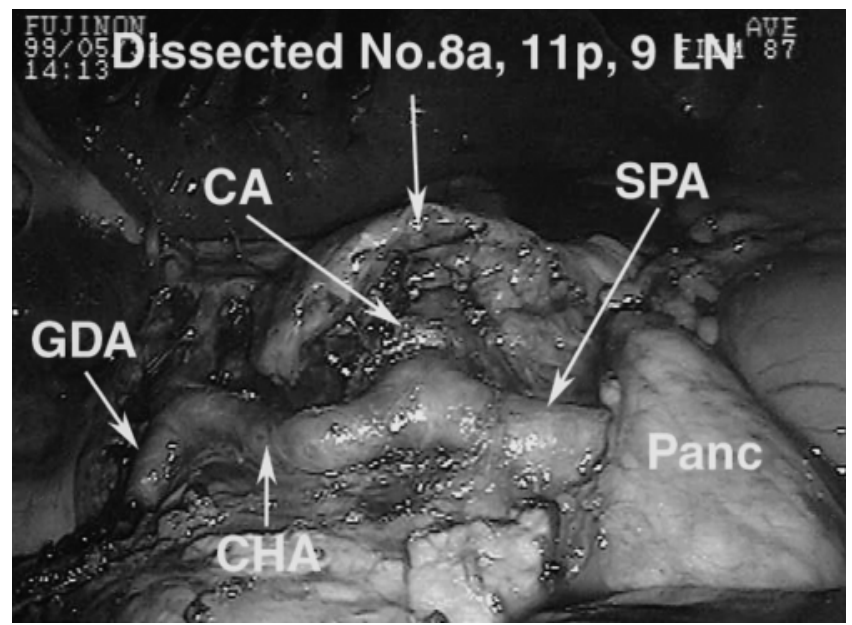

Fig. 4. Dissection of no. $8 \mathrm{a}, 9$, and $11 \mathrm{p}$ lymph nodes. $G D A$, Gastroduodenal artery; $C A$, celiac artery; $S P A$, splenic artery; $C H A$, common hepatic artery; panc, pancreas; $L N$, lymph node

placed in a specimen-bag (Endocatch II; United States Surgical), and temporarily left in the right subphrenic space or pelvic cavity.

In the reconstruction, the intracorporeal anastomosis was performed with a Roux-en-Y gastrojejunostomy. This anastomotic procedure was carried out with a functional end-to-end anastomotic technique, using the endoscopic stapling devices.

The left lower trocar wound was extended to 3.0$3.5 \mathrm{~cm}$ in length and the plastic bag containing the resected stomach and resected lymph nodes was retrieved. Two drains were placed through the bilateral trocar wounds.

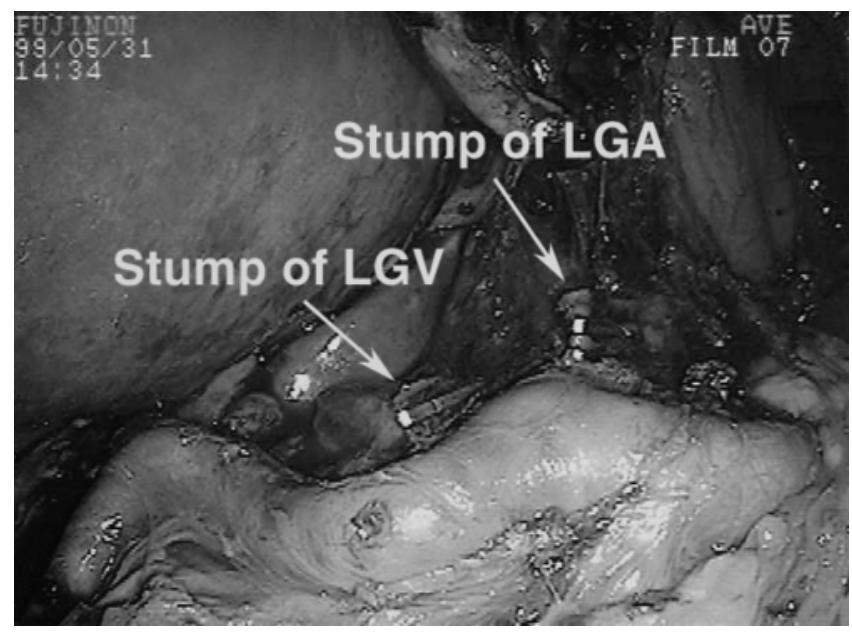

Fig. 5. Exposure and division of the left gastric artery $(L G A)$. $L G V$, Left gastric vein

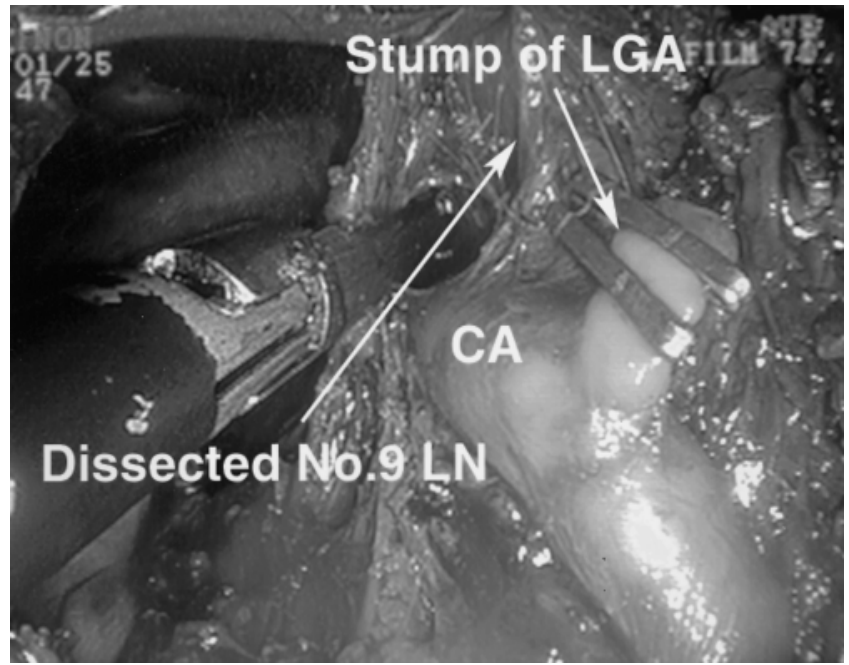

Fig. 6. Addition of no. 9 lymph node dissection. $L G A$, Left gastric artery; $C A$, celiac artery; $L N$, lymph node

\section{Results}

We performed completely laparoscopic distal gastrectomy with extraperigastric lymph node dissection in 12 patients: 11 with early gastric cancer, and 1 with malignant lymphoma. In all patients, the procedure was completed without conversion to open surgery. There were no mortalities in this series. One patient experienced an anastomotic stricture, which was treated with endoscopic balloon dilatation. Another patient suffered from Roux-en-Y stasis syndrome [9], which was alleviated by the oral administration of erythromycin. Otherwise, the patients had no significant complications. 


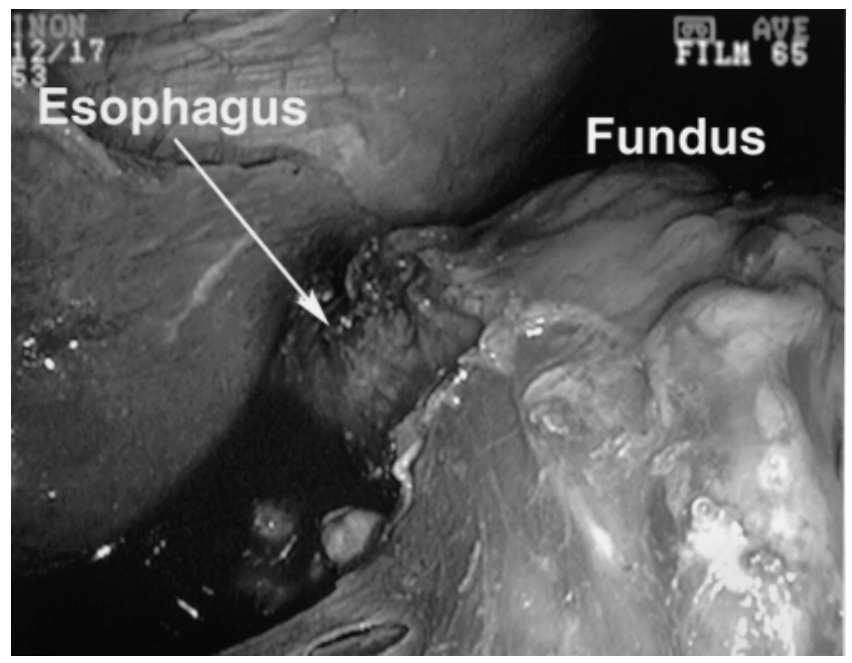

Fig. 7. Dissection of the no. 1 lymph nodes along with skeletonization of the upper third of the lesser curvature

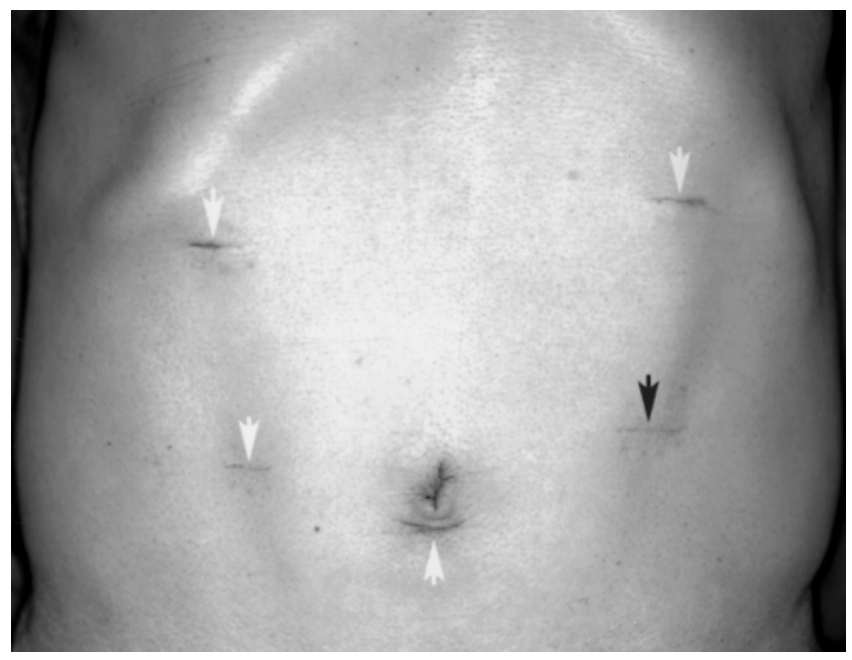

Fig. 8. Surgical wounds. Photograph shows surgical wounds 3 months after the surgery. White arrows, Trocar wounds; black arrow, extended wound (specimen was removed through this wound)

The average operative time was 409.7 min (range, $272-517 \mathrm{~min}$ ), which was significantly longer than that for the same open surgery. The average blood loss was $76.3 \mathrm{~g}$ (range, 22-235g), and only autologous blood transfusions were required. The average number of dissected lymph nodes per patient was 46.4 (range, 25-74). This was not significantly different from the number of nodes dissected using the open surgical technique. The average maximum length of the operative wound was $3.3 \mathrm{~cm}$ (range, $3.0-3.5 \mathrm{~cm}$ ) (Fig. 8).

Postoperative ambulation (average, 1.8 days; range, 1-3 days), and oral feeding (average, 4.2 days; range,
3-7 days) were both initiated more quickly after the laparoscopic procedure. The number of times the patients requested analgesic (average, 2.1 times; range, 0-3 times) was also less than with the corresponding open procedure.

\section{Discussion}

Laparoscopy - assisted distal gastrectomy with D1 + no. 7 lymph node dissection has been performed for mucosal gastric cancer when the lesions are not suitable for endoscopic mucosal resection or laparoscopic local resection due to tumor size, depth, and location $[3,4]$. To employ laparoscopic surgery for submucosal gastric cancer, extraperigastric lymph node dissection is necessary [10]. Because of this requirement, laparoscopyassisted extraperigastric lymph node dissection via minilaparotomy has been performed recently [11, 12]. However, in our experience, the extraperigastric lymph node dissection via minilaparotomy requires an incision $7-10 \mathrm{~cm}$ in length (depending on the patient's body habitus), bringing into question whether or not laparoscopy-assisted surgery is really less invasive. Therefore, to minimize the invasiveness of the procedure, we attempted completely laparoscopic extraperigastric lymph node dissection in patients with distal gastrectomy. This modified operative technique required only small incisions (the average maximum length being $3.3 \mathrm{~cm}$ ).

In the laparoscopy-assisted surgery, dissections of the no. 8a and 9 lymph nodes were performed via minilaparotomy. Again, it is our belief that such lymph node dissections are accomplished more easily and more safely with laparoscopy. In short, the laparoscopic view is visually superior to the direct view via minilaparotomy, and the laparoscopic instruments and the surgeon's skill have been improved.

In this surgical series, completely laparoscopic dissection of extraperigastric lymph nodes was technically feasible, had an acceptable complication rate, and had a curability similar to that with open surgery.

The advantages of the completely laparoscopic approach are a more rapid return to ambulation and oral feeding, and less postoperative pain. However, in order to investigate whether our procedure is actually less invasive, a study of immune response mediators (for example, interleukin-6 and tumor necrosis factor) and their concentrations after each of the various procedures is necessary $[13,14]$.

In summary, completely laparoscopic distal gastrectomy with extraperigastric lymph node dissection for gastric malignancies is as safe and reliable as open surgery. In the future, laparoscopic surgery may be employed in the treatment of patients with more advanced 
disease and may become standard procedure for gastric malignancies.

\section{References}

1. Kitano S, Iso Y, Moriyama M, Sugimachi K. Laparoscopicassisted Billroth I gastrectomy. Surg Laparosc Endosc 1994;2: 146-8.

2. Kuo WH, Lee WJ, Chen CN, Yuan RH, Yu SC. Laparoscopic subtotal gastrectomy with lymphadenectomy in a patient with early gastric cancer. J Formos Med Assoc 1998;97:127-30.

3. Nagai $Y$, Tanimura $H$, Takifuji $K$, Kashiwagi $H$, Yamoto $H$, Nakatani Y. Laparoscope-assisted Billroth I gastrectomy. Surg Laparosc Endosc 1995;5:281-7.

4. Kitano S, Shimoda K, Miyahara M, Shiraishi N, Bandoh T, Yoshida T, et al. Laparoscopic approaches in the management of patients with early gastric carcinomas. Surg Laparosc Endosc 1995;5:359-62.

5. Uyama I, Ogiwara H, Takahara T, Furuta T, Kikuchi K, Iida S. Laparoscopic minilaparotomy Billroth I gastrectomy with extraperigastric lymphadenectomy for early gastric cancer using an abdominal wall-lifting method. J Laparoendosc Surg 1995;5: $181-7$.
6. Carios BL, Xavier BV, Marco C, Mato $\mathrm{R}$, Ruggiero $\mathrm{R}$. Laparoscopic Billroth II distal subtotal gastrectomy with gastric stump suspension for gastric malignancies. Am J Surg 1996;171: 289-92.

7. Japanese Research Society for Gastric Cancer. Japanese classification of gastric carcinoma. First English ed. Tokyo: Kanehara; 1995.

8. Japanese Gastric Cancer Association. Japanese classification of gastric carcinoma. 2nd English ed. Gastric Cancer 1998;1:1024.

9. Gustavsson S, Ilstrup DM, Morrison P, Kelly KA. Roux-Y stasis syndrome after gastrectomy. Am J Surg 1988;155:490-494.

10. Namieno T, Kito K, Higashi T, Sato N, Uchino J. General pattern of lymph node metastasis in early gastric cancer. World J Surg 1996;20:996-1000.

11. Hayashi K, Munakata Y. Laparoscopic assisted distal gastrectomy with regional lymphadenectomy. Surg Endosc 1998;12:558.

12. Hoshino T, Hashimoto D, Takahashi T. Laparoscopic-assisted gastrectomy with systematic lymphadenectomy: can such a complicated procedure be performed laparoscopically alone. Surg Endosc 1998;12:559.

13. Dinarello CA. Interleukin-1. Rev Infect Dis 1984;6:51-95.

14. Takahara T, Uyama I, Ogiwara H, Furuta T, Iida S. Inflammatory responses in open versus laparoscopic herniorrhaphy. $\mathrm{J}$ Laparoendosc Surg 1995;5:317-26. 